4. Baron TH. Direct percutaneous endoscopic jejunostomy through a mature gastrostomy tract. Gastrointest Endosc. 2002;56:946-947.

5. Adler DG, Gostout CJ, Baron TH. Percutaneous transgastric placement of jejunal feeding tubes with an ultrathin endoscope. Gastrointest Endosc. 2002;55:106-110

6. Nishiwaki S, Araki H, Shirakami Y, Kawaguchi J. Direct percutaneous endoscopic jejunostomy using a transgastrostomic endoscope in patients with previous endoscopic gastrostomy. Endoscopy. 2009;41(S 02),E36-E37.

\section{Primary Aortoenteric fistula : An alarming entity warranting early diagnosis}

\section{Introduction}

Aortoenteric fistulas (AEF) are communications between the aorta and a loop of the bowel. Primary AEF is a rare and challenging entity and requires awareness and a vigilant approach to reach the diagnosis. Autopsy findings suggest that the incidence of primary AEF range between 0.04\% and $0.07 \% .{ }^{1}$ Despite advances in technology, an AEF is difficult to diagnose and treat and is associated with high mortality (65-100\%) and morbidity. ${ }^{2}$ The radiologist plays a pivotal role in reaching an accurate and precise diagnosis of AEF. Imaging tools such as ultrasound and multidetector contrast enhanced computed tomography (CECT) helps in making an accurate diagnosis and to differentiate it from other inflammatory mimickers. We describe a case report of a 46 year old woman presenting to the emergency with shock and a history of melena and was subsequently accurately diagnosed to have an aortoenteric fistula. The objective of presenting this case report is to discuss the radiological findings of primary aortoenteric fistula in a patient presenting with acute hemodynamic instability.

\section{Case Report}

A 46 year old woman was referred to the emergency with melena and shock. She had a history of sudden abdominal pain, vomiting and weakness for one day. She had a past history of on and off fever for the last 1 year with loss of weight and appetite. There was no history of previous abdominal vascular surgery. On systemic examination, the patient was found to have pallor, feeble pulse and was hemodynamically unstable with a blood pressure (BP) of $80 / 50$, heart rate (HR) of 138 beats per minute, $\mathrm{C}$ reactive protein (CRP) of 96 and hemoglobin $(\mathrm{Hb})$ of $6.2 \mathrm{gm} \%$. The patient had a further drop in hemoglobin to a $\mathrm{Hb}$ of $3.7 \mathrm{gm} \%$ within 12 hours. To search for the cause of the sudden drop in hemoglobin and patient's worsening condition, a multidetector contrast enhanced computed tomography (CECT) was acquired on a 64 slice scanner (GE healthcare) by giving $100 \mathrm{ml}$ of iv contrast $(300 \mathrm{mg}$ /l) at the rate of $4.0 \mathrm{ml} / \mathrm{sec}$ and arterial phase images were acquired by bolus tracking followed by portal venous and delayed phase at 65 and 180 seconds respectively. However, despite being on resuscitative fluids and triple inotropes, the patient progressed to become severely hypotensive and had a cardio respiratory arrest from which she could not be revived even after cardiopulmonary resuscitation. The relatives of the patient didn't give consent for the autopsy.

Multidetector CECT revealed the presence of an active contrast extravasation from the infrarenal aorta in the left paraaortic location forming a large hematoma measuring $8.2 \times 7 \times 9.2 \mathrm{~cm}$. Multiple air foci were seen adjacent to the aorta and in the hematoma, with direct communication with the fourth part (D4) of the duodenum (Figure 1a,b). A breech of $2.4 \mathrm{~cm}$ was seen in the posterior wall of the D4 segment of the duodenum (Figure 2a). The delayed images revealed the presence of contrast in the proximal jejunal loops (Figure $2 \mathbf{b}$ ). The abdominal aorta, from the level of origin of the superior mesenteric artery to its bifurcation shows circumferential asymmetric mural thickening with irregular luminal narrowing (Figure 3a). Additional findings included chronic thrombosis of the left renal artery with atrophic left kidney and chronic thrombosis of the left common iliac artery and right external iliac artery (Figure $\mathbf{3 b}, \mathbf{c}$ ).

\section{Discussion}

An aortoenteric fistula (AEF) is the abnormal 


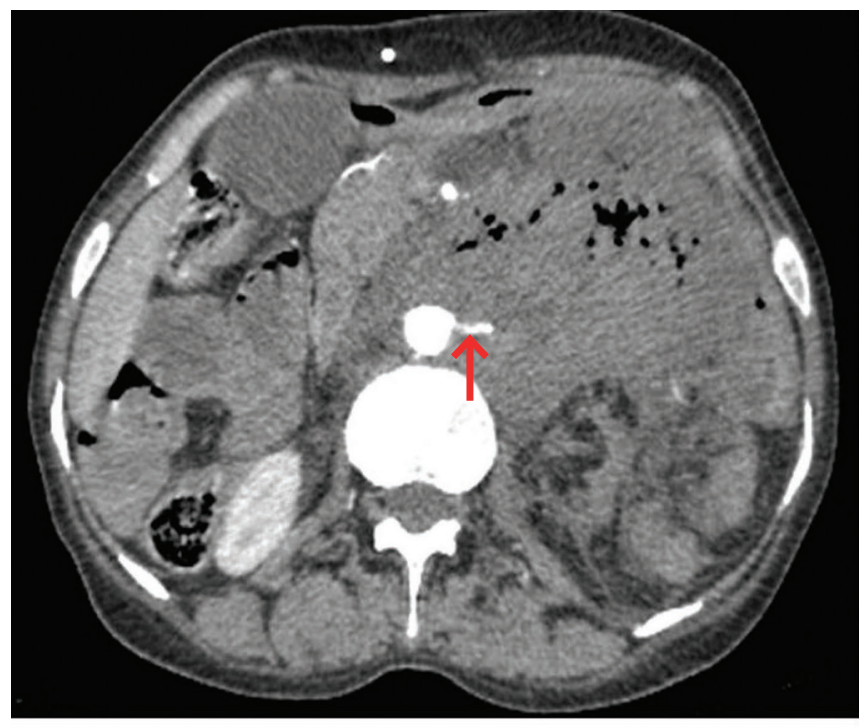

a

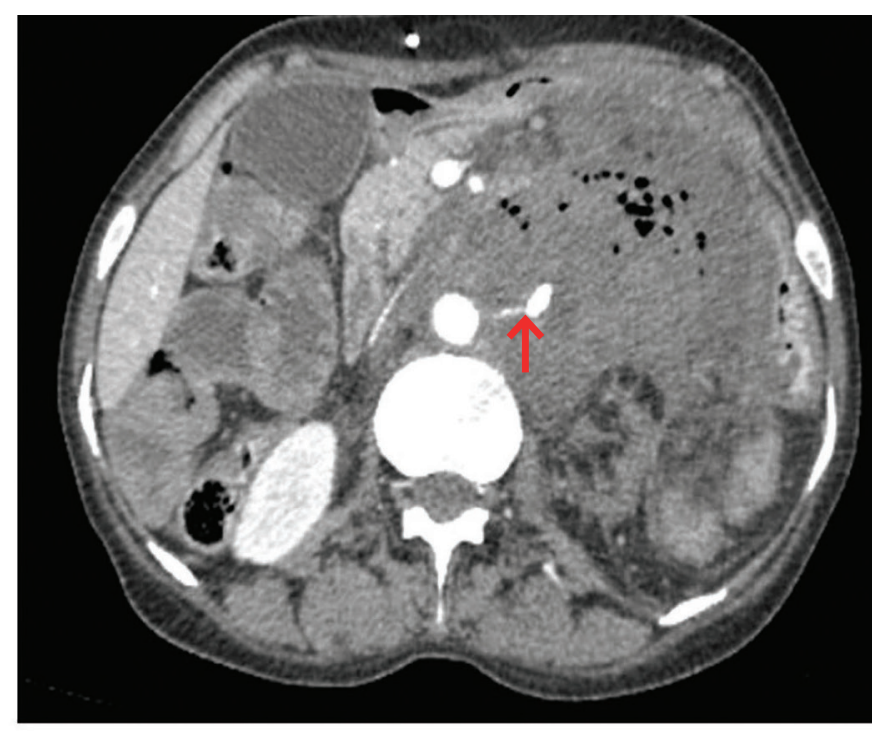

b

Figure 1a and b: Axial contrast enhanced CT arterial phase shows active contrast extravasation from the infrarenal aorta $\uparrow$ and air foci in the large adjacent hematoma.

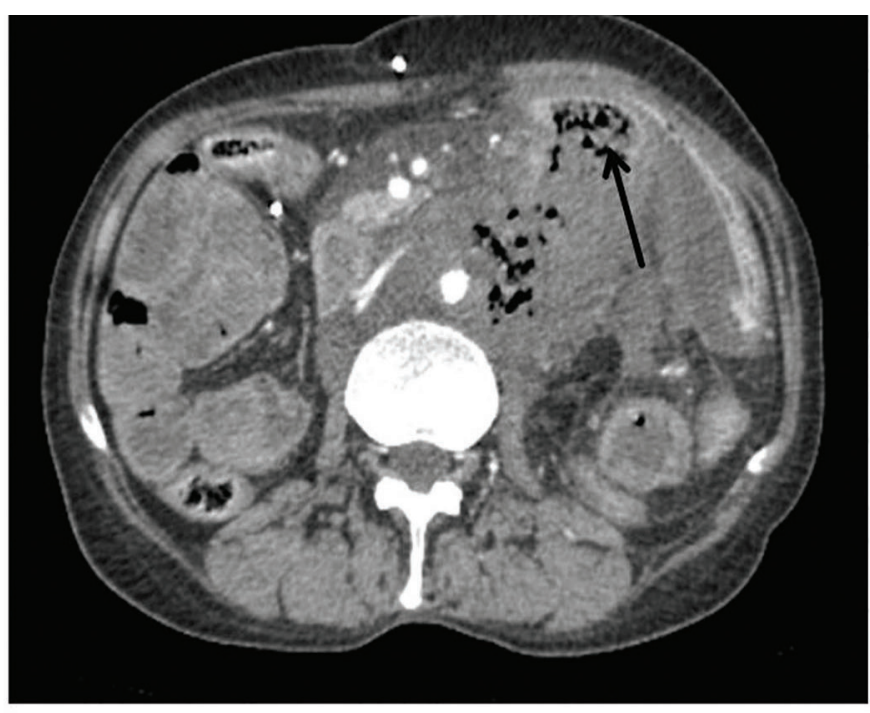

a

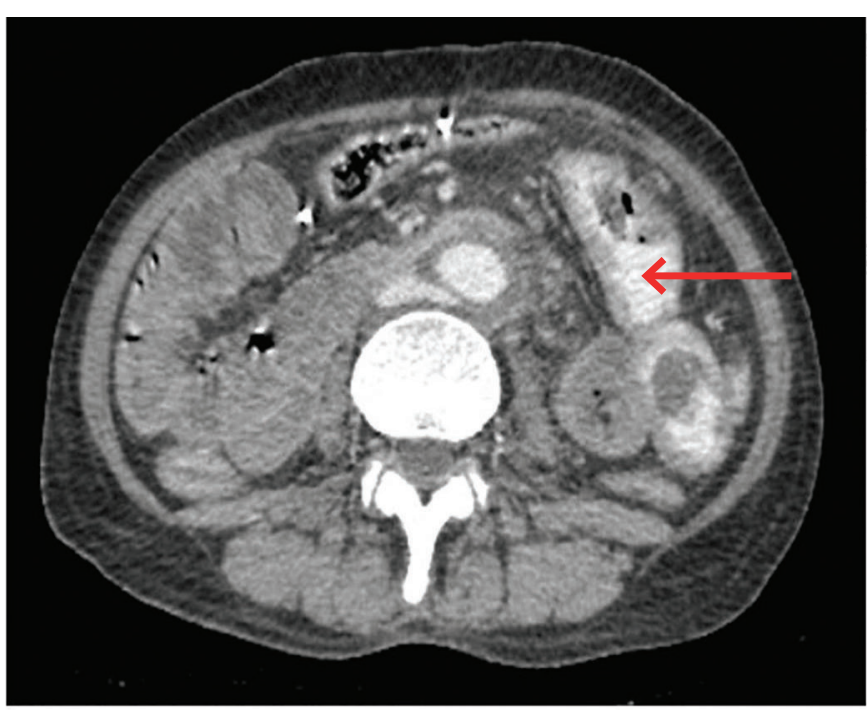

b

Figure 2a: Axial contrast enhanced CT venous phase shows breech in the inferomedial wall of the duodenum (arrow); b Delayed images revealed contrast in the proximal jejunal loops $\longleftarrow$

communication between the aorta and the bowel loop. It can be primary or secondary, based on the history of abdominal aortic aneurysm (AAA) repair and the presence of prosthetic materials. Primary AEFs are rare and challenging entities and require awareness and a vigilant approach for its diagnosis. Most primary AEFs are seen in the duodenum (60\%), arising in the 3rd and 4 th part of the duodenum due to close proximity and direct contact with the abdominal aorta. The other $40 \%$ are associated with other loops of small and large bowel such as jejunum (12\%), ileum (18\%), cecum (8\%), and appendix (4\%). ${ }^{3}$ Approximately $99 \%$ of primary AEFs 


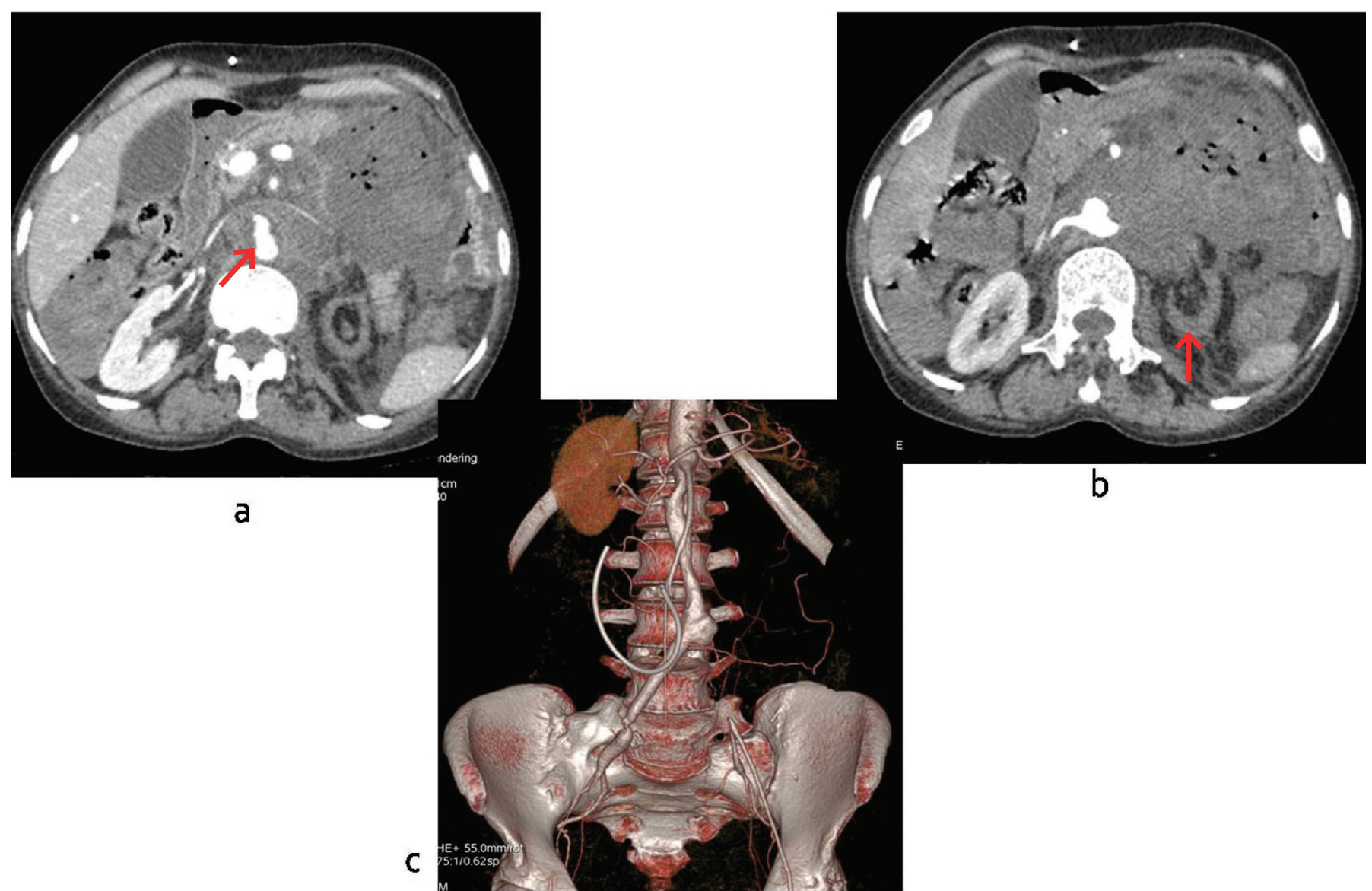

Figure 3a: Axial contrast enhanced CT arterial phase shows irregular mural thickening of aorta $\nearrow$; $3 \mathrm{~b}$ : Axial contrast enhanced CT arterial phase shows non opacification of the left renal artery with atrophic left-kidney $\uparrow ; 3 \mathrm{c}$ : Volume rendered tomographic (VRT) coronal image depicts non visualisation of the left common iliac artery and right external iliac artery.

have been estimated to arise from an abdominal aortic aneurysm, mostly atherosclerotic. Remaining cases were caused by radiation, pancreatic carcinoma, ulcers, diverticulitis, appendicitis and metastases. ${ }^{4}$ The most common cause is a ruptured atherosclerotic abdominal aortic aneurysm that occurs secondarily to the pressure of the pulsating aneurysm on the adjacent bowel loop wall resulting in pressure necrosis.

Among the wide armamentarium offered by radiology, the multidetector contrast enhanced helical CT scan is the first line and the most preferred imaging modality of choice with sensitivity of $40-90 \%$ and specificity of 33-100\%. ${ }^{5}$ Other studies such as upper GI endoscopy, angiography and nuclear studies such as scintigraphy with SPECT using radiotracers gallium 67 citrate, $111 \mathrm{In}$ labeled white blood cells or $99 \mathrm{mTc}$ hexametazime, can be helpful. However CT is by far the best modality owing to its widespread availability, short acquisition time and high resolution. The direct signs are the presence of ectopic gas around and within the aorta and the intravenous contrast in the gastrointestinal tract. Indirect signs include adjacent bowel wall thickening, disruption of the aortic fat cover and aortic wall, tethering of bowel loop to the anterior wall of the aorta and retroperitoneal hematoma or hematoma within bowel wall or lumen. Based on the above described signs, the diagnosis of a primary aortoenteric fistula was made in our patient. The list of differential diagnosis includes retroperitoneal fibrosis, infected aortic aneurysm, infectious aortitis, perigraft infection without fistulisation. The incidence of mortality in the untreated primary AEF is almost $100 \%$, thus it warrants urgent treatment. The survival after surgery ranges from $18 \%$ to $93 \%$, depending on the hemodynamic instability of the patient and experience of the surgeon. ${ }^{6}$ Thus the radiologist plays a pivotal role in making an early diagnosis of this lethal entity. 


P NARANJE ${ }^{1}$
U GORSI $^{1}$
L GUPTA $^{1}$
A BHALLA ${ }^{2}$
N KHANDELWAL ${ }^{1}$
Correspondence: Priyanka Naranje ${ }^{1}$
Post Graduate Institute of Medical Education and
Research, Chandigarh, India.
E mail: priyanka11sh@gmail.com

\section{References}

1. Voorhoeve R, Moll FL, de Letter JA, Bast TJ, Wester JP, Slee PH. Primary aortoenteric fistula: report of eight new cases and review of the literature. Ann Vasc Surg. 1996;10:40-8.

2. Hughes FM, Kavanagh D, Barry M, Owens A, MacErlaine DP, Malone DE. Aortoenteric fistula: a diagnostic dilemma. Abdom Imaging. 2007;32:398402.

3. Champion MC, Sullivan SN, Coles JC, Goldbach M, Watson WC. Aortoenteric fistula. Incidence, presentation recognition, and management. Ann Surg. 1982;195:314-7.

4. Gad A. Aortoduodenal fistula revisited. Scand J Gastroenterol Suppl. 1989;167:97-100.

5. Vu QD, Menias CO, Bhalla S, Peterson C, Wang LL, Balfe DM. Aortoenteric fistulas: CT features and potential mimics. Radiographics. 2009;29:197-209.

6. Kavanagh DO, Dowdall JF, Younis F, Sheehan S, Mehigan D, Barry MC. Aorto-enteric fistula: changing management strategies. Ir J Med Sci. 2006;175:40-4.

\section{Rare Cause Of Recurrent Acute Pancreatitis Due To Leiomyosarcoma}

\section{Introduction}

Recurrent pancreatitis is a common clinical problem encountered by the gastroenterologist. Its common causes are gall bladder stones, biliary microliths, alcohol, early presentation of chronic pancreatitis and metabolic causes like hypercalcemia and hypertriglyceridemia. Rarely, it can be caused by pancreatic ductal obstruction due to an ampullary or pancreatic neoplasm. Recently, in one of our patients who presented with recurent acute pancreatitis, we found the cause to be a leiomyosarcoma, which is very rare in the pancreas and has not been described previously in literature as a cause of recurrent pancreatitis.

\section{Case Report}

A 45-year old lady presented in February 2012, with the complaint of recurrent abdmoinal pain of 5 years duration. She had no past history of any alcohol intake or of gall stone disease. The pain was epigastric in location with radiation to the back, and was associated with abdominal distension, obstipation and bilious vomiting without any history of jaundice, fever, gastrointestinal bleed, decreased urine output or dyspnoea. During the attacks of pain, levels of serum amylase and lipase would rise to more than 3 to 5 times the upper limit of normal. Contrast enhanced computed tomography done during the initial episode of pain revealed a dilated main pancreatic duct and a bulky pancreas without any evidence of pancreatic parenchymal calcification or mass in pancreas. Based on the biochemical and imaging findings, the patient was initially diagnosed as a case of chronic pancreatitis with recurrent episodes of acute exacerbations. Each episode of pain was treated with parenteral analgesics. The pain would last for two to five days and the patient was apparently healthy in between the atacks. The patient had had multiple episodes of pain over the previous 5 years, with a frequency of one or two episodes per year.

During the last such episode in February 2012, she presented to our hospital with the complaint of significant weight loss and persistent pain for more than 4 weeks. Her physical examination was unremarkable except for her low body mass index. Contrast enhanced CT scan revealed dilated main pancreatic duct with a $3 \mathrm{~cm}$ cyst in the head of the pancreas without any pancreatic calcification (Figure 1). On account of change 